\title{
Establishment of Standard Human Blood Vessel Model Based on Image Registration and Fitting Technology
}

\author{
Dinghao Luo ${ }^{1}$ Junxiang $\mathrm{Wu}^{1} \cdot \mathrm{Ning} \mathrm{Wang}^{1} \cdot$ Lei Wang $^{1} \cdot \mathrm{Kai}_{\mathrm{Xie}}{ }^{1} \cdot$ Songtao $\mathrm{Ai}^{2} \cdot$ Lingjie Fu $^{1} \cdot$ Yongqiang Hao $^{1}$. \\ Wen $\mathrm{Wu}^{1}$
}

Received: 7 January 2021 / Accepted: 29 December 2021 / Published online: 25 January 2022

(c) The Author(s) 2022

\begin{abstract}
Purpose The blood vessel gives key information for pathological changes in a variety of diseases. In view of the crucial role of blood vessel structure, the present study aims to establish a digital human blood vessel standard model for diagnosing blood vessel-related diseases.

Methods The present study recruited eight healthy volunteers, and reconstructed their bilateral upper extremity arteries according to CTA. The reconstructed vessels were segmented, registered, and merged into a bunch. After being cut by continuous cut planes, the dispersion of the blood vessel bunches on each cut plane were calculated.

Results The results demonstrated that the middle segment of the brachial artery, the proximal segment of the ulnar artery, and the middle and distal segments of the radial artery had a low degree of dispersion. A standard blood vessel model was finally established by the integral method using the low-dispersion segments above. The accuracy of the standard blood vessel model was also verified by an actual contralateral vessel, which revealed that the deviation between the model and the actual normal contralateral brachial artery was relatively small.

Conclusion The structure of the model was highly accordant with the real ones, which can be of great help in evaluating the blood vessel changes in blood vessel-related diseases, bone and soft-tissue tumors, and creating accurate surgical plans.
\end{abstract}

Keywords Blood vessel $\cdot$ Standard model $\cdot$ Image registration and fitting $\cdot$ CTA reconstruction

\section{Introduction}

Blood circulation is of critical significance for life maintenance. It is extensively involved in various metabolic functions, such as oxygen and carbon dioxide exchange, digestion and absorption, and urine formation, to name just

Wen $\mathrm{Wu}$

Wen_Wu_L@163.com

Lingjie Fu

Lingjie_Fu_L@163.com

Yongqiang Hao

Yongqiang_Hao_L@163.com

1 Department of Orthopaedic Surgery, Shanghai Key Laboratory of Orthopaedic Implants, Shanghai Ninth People's Hospital, Shanghai Jiaotong University School of Medicine, Shanghai 200011, China

2 Department of Radiology, Shanghai Ninth People's Hospital, Shanghai Jiaotong University School of Medicine, Shanghai 200011, China a few. The blood vessel is the basis of blood circulation, and the pathological changes of blood vessel structure can cause crucial damage of tissues, resulting in a variety of diseases [1-4]. The blood vessel structure is unquestionably of great significance for the diagnosis, evaluation, and treatment of diseases, and the high incidence of angiopathy cannot be ignored. Infantile hemangioma is the most common benign tumor of infancy, with a incidence of $4.5 \%$, which adequately depicts the perniciousness of blood vessel-related diseases [5].

Computed tomography angiography (CTA), magnetic resonance angiography (MRA), X-ray arteriography, Doppler blood vessel ultrasound, and other related technologies provide a pivotal imaging basis for the analysis of blood vessel structure, and consequently play a crucial diagnostic role in diseases involving renal vessels, coronary vessels, liver vessels, and so on [6-8]. However, there is still a considerable gap between the collected imaging data and the actual lesions. The limitations of two-dimensional (2D) image data lead to the deficiency of stereoscopic and intuitive 
recognition, which significantly highlights the necessity to transform the traditional planar anatomical information into three-dimensional (3D) structure.

By the 3D reconstruction of $2 \mathrm{D}$ image data, the structure and scope of blood vessels in the lesion region can be efficiently elucidated. Whereas, to optimize the diagnostic accuracy of blood vessel relevant diseases, it is believed to be of vital importance to compare the reconstructed blood structure of patients with a representative standard blood vessel reference. The idea of using symmetrical blood vessels on the contralateral side of the lesion as a reference was found to be useful, but the effect of this was clearly limited when dealing with lesions that occur in the asymmetric blood vessels. In addition, for the vast majority of patients undergoing primary disease screening, an extra CTA on the healthy side is not feasible due to increased operation time, trauma, cost, and other issues. Thus far, there was no representative reference standard for the 3D structure and location of blood vessels, which further resulted in difficulties in unified computer processing and surgical planning. Based on the foregoing situation, this study analyzed and integrated the reconstructed CTA data of eight volunteers based on image registration and fitting technology. After investigating the appropriate blood vessel segments, an innovative registration and fitting methodology has been preliminarily proposed to establish a 3D digital standard blood vessel model with verified accuracy. Such a model can provide a practical and promising reference for the diagnosis and treatment of blood vessel-related diseases.

\section{Materials and Methods}

\subsection{Volunteer Recruitment and CTA Data Acquisition}

The flow diagram of the whole process of the study was shown in Fig. 1A. For this study, a total of eight healthy adult volunteers ( $28.4 \pm 8.8$ years, range $22-49$ years, eight men) with relatively consistent height and weight were recruited. The demographics of the group are provided in Table 1. During the examination, the volunteers were instructed to follow the standard anatomical position as much as possible, to make the results more uniform. Their CTA image data of bilateral upper extremity arteries were collected using the SOMATOM definition flash CT machine (Siemens Healthcare, Germany). The scanning range was from head to foot, the scanning voltage was $120 \mathrm{kV}$, and the layer thickness was $1.5 \mathrm{~mm}$. The obtained CTA data of all eight subjects were stored in DICOM 3.0 standard data format. To obtain clearer and more accurate arterial structures, the scanning was processed in the arterial phase. During CTA, the volunteer was in the supine position.
A
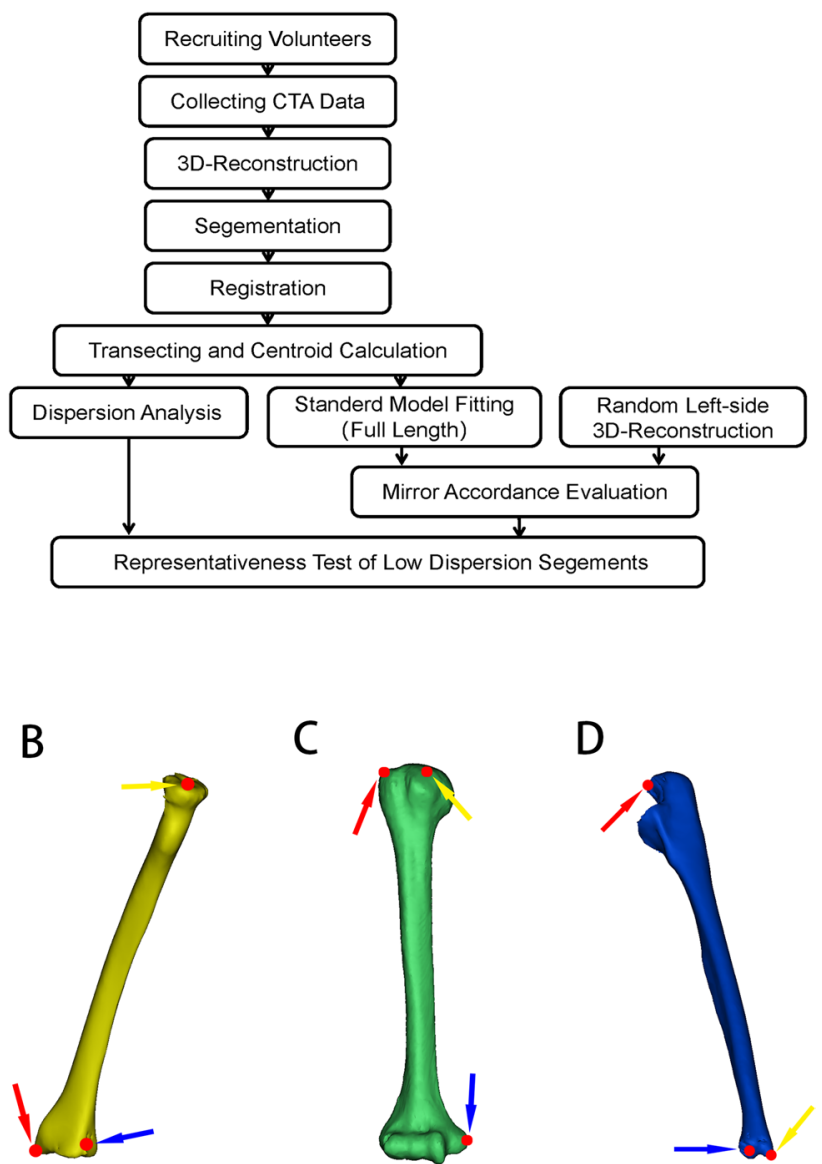

C
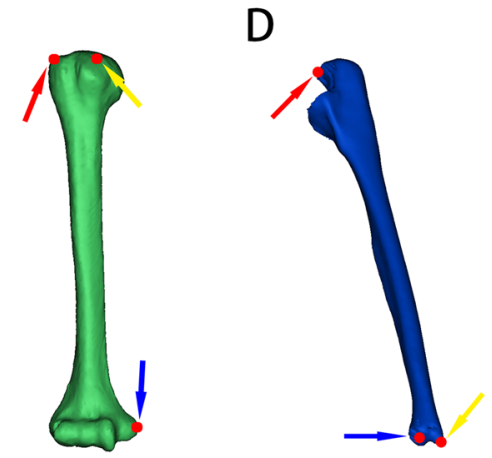

Fig. 1 Schematic diagram of registration marker points on bones. A The flow diagram of the whole process of the study. B The three marker points of the radius, with the red, yellow, and blue arrows showing the styloid process of the radius, center of the head of the radius, and volar margin of the ulnar notch of the radius; $\mathbf{C}$ the three marker points of the humerus, with the red, yellow, and blue arrows showing the upper lateral side of the large nodule, the upper lateral side of the small nodule, and the tip of the inner epicondyle; $\mathbf{D}$ the three marker points of the ulna, with the red, yellow, and blue arrows showing the tip of the olecranon, the styloid process of the ulna, and the center of the carpal joint surface of the ulna

Table 1 Volunteer demographics $(n=8)$

\begin{tabular}{ll}
\hline Age (years) & $28.4 \pm 8.8$ \\
Male & $8 / 8(100 \%)$ \\
Height $(\mathrm{cm})$ & $172.3 \pm 5.9$ \\
Weight $(\mathrm{kg})$ & $68.3 \pm 9.8$ \\
BMI & $27.8 \pm 7.5$ \\
\hline
\end{tabular}

The present study was based on the ethical principles of the 1975 Declaration of Helsinki. The protocol of this study was approved by the local ethics committee prior to recruitment of volunteers, and all individuals involved in the study had written informed consent. 


\subsection{Reconstruction and Segmentation of Bone and Blood Vessel}

The CTA data of eight subjects were analyzed and processed by Mimics Medical 20.0 (Materialise, Belgium). The regions of bone were divided by threshold 150-250 HU. The corresponding 3D models were further reconstructed from the divided bone $\mathrm{CT}$ region.

The borders of arteries were completely divided according to the CTA image data. The 3D blood vessel structure of the eight subjects on the right side were further reconstructed. A morphological interpolation method was used to calculate the shape of bone and blood vessels between the adjacent CT slices. After the 3D blood vessel model was reconstructed, the bifurcation of the radial and ulnar arteries was chosen as a demarcation point, and the blood vessels were further divided into brachial artery, ulnar artery, and radial artery.

\subsection{Upper Extremity Artery Registration}

After being divided into brachial artery, ulnar artery, and radial artery, the reconstructed blood vessel structure data of the right upper extremity arteries were transformed into STL data together with the upper extremity bone structure. The 3D reconstruction structure of the volunteers was further processed by Mimics 3-matic Medical 12.0 (Materialise, Belgium) to conduct upper extremity artery registration.

The process of bone and blood vessel registration was based on the point-to-point registration function in the Mimics 3-matic Medical 12.0 software. The bone structures of all eight subjects were divided into the humerus, ulna, and radius, and three marker points on each bone were selected by senior physicians (Fig. 1B-D). Specifically, the three marker points of the radius are the styloid process of the radius, the center of the head of the radius, and the volar margin of the ulnar notch of the radius. The three landmarks of the humerus are the upper lateral side of the small nodule, the upper lateral side of the large nodule, and the tip of the inner epicondyle. The three landmarks of the ulna are the tip of the olecranon, the styloid process of the ulna, and the center of the carpal joint surface of the ulna. A volunteer whose posture was relatively consistent with the anatomical posture during $\mathrm{CT}$ acquisition was selected from the eight volunteers as the standard reference, and each bone of the other seven volunteers was moved under the instruction of the software to align the position of the marker points with the standard reference.

In the process of bone registration, the brachial artery, ulnar artery, and radial artery also moved together with the corresponding bone to achieve blood vessel registration. Specifically, the brachial artery corresponded to the humerus, the ulnar artery corresponded to the ulna, and the radial artery corresponded to the radius. The relative position relationship between the blood vessel and its corresponding bone remains unchanged in the registration process. After registration, the position and direction of bones and blood vessels were unified, forming a blood vessel bundle.

\subsection{Dispersion Analysis of Different Segments of Blood Vessel Bundle}

The bundle was further transected by consecutive cut planes with 5-mm intervals. Because the initial point of each vessel was different after registration, the cut planes started from the position that consisted of all eight vessels in the blood vessel bundle and continued to the radial artery and the ulnar artery. According to the interval of $5 \mathrm{~mm}$, the whole length of the brachial artery was divided into 73 cut planes, and the ulnar and radial arteries, as a whole, were divided into $31 \mathrm{cut}$ planes. According to the direction of blood vessels, among the cut planes of the brachial artery, the 1st to the 13th were vertical cut planes, the 14 th to the 20th were oblique cut planes, and the 21 st to 73 rd were horizontal cut planes. All cut planes of the ulnar and radial arteries were horizontal.

The orthogonal coordinate system was established on each cut plane with the intercut line of the cut plane and the sagittal plane as the Y-axis and the intercut line of the cut plane and the coronal plane as the $\mathrm{X}$-axis. On each cut plane, each blood vessel left a section, whose shape was mostly irregular, but almost circular. Employing the centroid calculation function of Mimics 3-matic Medical 12.0 (Materialise, Belgium), the centroids of the sections were obtained. After the eight centroids of the eight vessels on a same cut plane were calculated, eight pairs of $\mathrm{X}$ and $\mathrm{Y}$ coordinates of centroids were recorded, and the standard deviations of $\mathrm{X}$ and $\mathrm{Y}$ were further calculated, to indicate the degree of dispersion of the blood vessel bundle on this cut plane. All cut planes that had a low degree of dispersion constituted the appropriate low-dispersion blood vessel segments that can be further used for fitting the standard blood vessel model.

\subsection{Fitting the Reconstruction of the Standard Blood Vessel Model and the Accordance Evaluation}

The average $\mathrm{X}$ coordinate and $\mathrm{Y}$ coordinate of eight centroids were calculated as the standard coordinate reference; the average area of eight irregular blood vessel sections were calculated as the standard area reference. A standard circle was created, with the standard coordinate reference being its center and the standard area reference being its circular area.

All the circles on cut planes were successively connected using the morphological interpolation method of Mimics 3-matic Medical 12.0 (Materialise, Belgium). Next, a curved 
irregular cylinder with varying directions and diameters was formed, which was regarded as the standard blood vessel model of the full length of the right upper limb artery.

In addition, after the CTA data of the left upper limb artery of each case was obtained, the 3D structures of the left upper limb artery were also reconstructed. After a simple mirror symmetry administration, according to the position of the bone, the calculated right side standard blood vessel model was registered to a random actual left upper limb artery. After registration, according to the consistency of the two vessels in the spatial location, the accordance of the standard vessel model was evaluated.

\section{Results}

\subsection{The Location and Direction of Blood Vessels Were Effectively Unified after Segmentation and Registration}

After 3D reconstruction, the CTA upper limb arteries and bone structures were clearly presented and morphologically conformed to the general anatomy. The humerus, ulna, radius and corresponding brachial artery, ulnar artery, and radial artery were properly divided (Fig. 2A).

The overall structures of the blood vessels in the eight subjects before and after segmentation and registration are respectively shown in Fig. 2B and C. It can be seen that after registration, the location of the eight blood vessels was relatively accordant and the direction was relatively consistent, forming a relatively neat blood vessels bunch, which can preliminarily represent the general structure trends of human blood vessels.

A

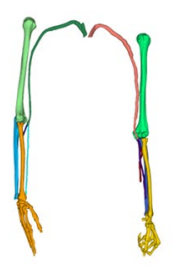

B

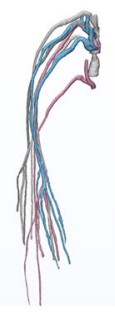

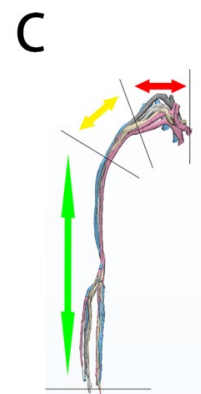

Fig. 2 Segmental registration and blood vessel bunch construction. A Schematic diagram of upper limb skeleton and blood vessel division; B the front view of three-dimensional blood vessel bunch before registration; $\mathbf{C}$ the front view of the three-dimensional blood vessel bunch after registration showing a more orderly vascular structure. The structure was further roughly directionally divided into horizontal, oblique, and vertical segments and marked with red, yellow, and green arrows, respectively

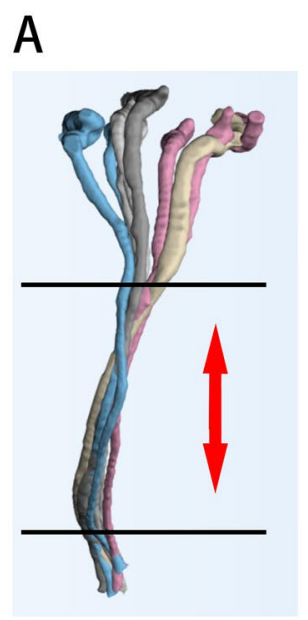

B
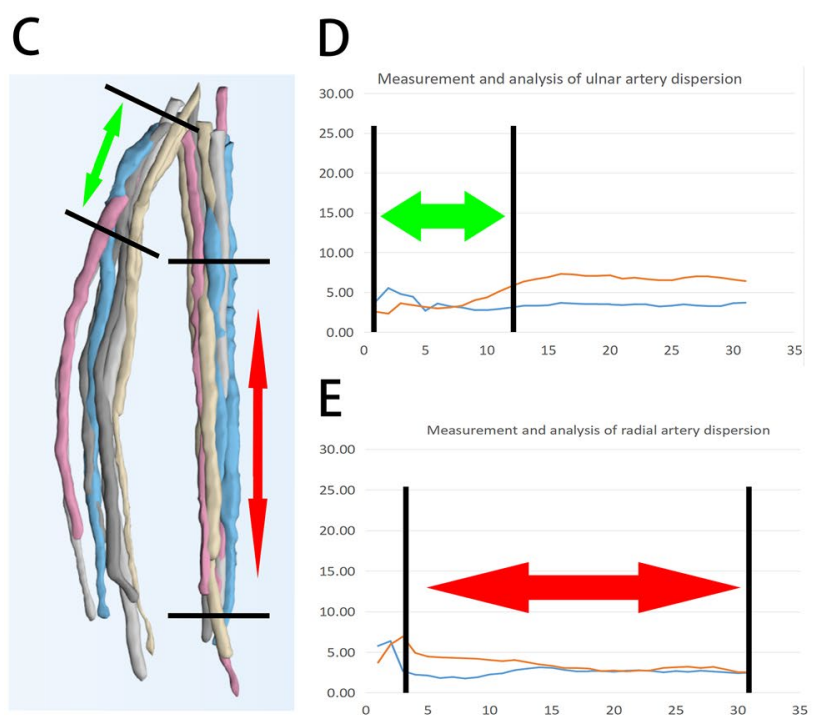

Fig. 3 Calculation of 3D blood vessel bundle dispersion. A Three-dimensional schematic diagram of brachial artery dispersion with the red arrow showing that the structure in the middle segment of brachial artery was relatively neat and consistent; $\mathbf{B}$ result of brachial artery dispersion with the blue line indicating the $\mathrm{X}$ coordinate and the orange line indicating the $\mathrm{Y}$ coordinate; $\mathbf{C}$ three-dimensional schematic diagram of radial and ulnar arteries dispersion with the green arrow showing that the structures in the proximal segment of the ulnar artery were relatively neat and consistent, and the middle and distal segments of the radial artery had similar promising results, shown by red arrows; $\mathbf{D}$ result of ulnar artery dispersion with the blue line indicating the $\mathrm{X}$ coordinate and the orange line indicating the $\mathrm{Y}$ coordinate; $\mathbf{E}$ result of radial artery dispersion with the blue line indicating the $\mathrm{X}$ coordinate and the orange line indicating the $\mathrm{Y}$ coordinate

\subsection{The Middle Segment of the Brachia Artery, the Proximal Segment of the Ulnar Artery, and the Middle and Distal Segments of the Radial Artery were Appropriate Low-dispersion Segments}

The dispersion degree results of each cut plane starting from the subclavian artery are shown in Fig. 3A and B. It 
Fig. 4 Accordance evaluation between the standard blood vessel model of the full length and the actual contralateral blood vessel. A The overall schematic diagram of standard model accordance, with pink indicating standard brachial artery model, gray indicating the standard ulnar artery and radial artery model, and red indicating the three-dimensional reconstruction of the actual blood vessel on the left side of a random volunteer. It can be seen that the calculated standard blood vessel model was highly accordant with the actual blood vessel in direction and structure. A cut plane was also marked in this figure; B a schematic diagram of sections on the aforementioned cut plane, with gray arrows indicating the section of calculated standard blood vessel model of the right side (circle), and red arrow indicating the actual blood vessel section (irregular), which demonstrated that these two were highly accordant in structure and direction; $\mathbf{C}$ the schematic diagram of the standard blood vessel model accordance evaluation of the brachial artery with the red arrow showing that the standard brachial artery model was highly accordant with the actual left brachial artery; D the 180-degree rotation schematic diagram of standard blood vessel model accordance evaluation of the brachial artery with the red arrow showing that the standard brachial artery model was still highly accordant with the actual left brachial artery; $\mathbf{E}$ the schematic diagram of the standard blood vessel model accordance evaluation of the ulnar artery and radial artery with the red and blue arrows showing that the standard radial artery model was highly accordant with the actual left radial artery, but the standard ulna artery model was not; F the 180-degree rotation schematic diagram of the standard blood vessel model accordance evaluation of the ulnar artery and radial artery with the red and blue arrows showing that the standard radial artery model was highly accordant with the actual left radial artery, but the standard ulna artery model was not

can be seen that at the right subclavian artery where the blood vessel bunch started, the distribution of the blood vessel location was relatively scattered, with a high degree of dispersion. With the cut planes moving toward the middle segment of the brachial artery, the relative distance of blood vessels gradually shortened, and the degree of dispersion gradually decreased and remained stable. Among them, in the range of the 19 th to the 70th cut plane, the dispersion degrees of $\mathrm{X}$ and $\mathrm{Y}$ were both less than $6 \mathrm{~mm}$ with the length being approximately $26 \mathrm{~cm}$, which significantly demonstrated that the distribution of blood vessels was relatively concentrated. When the continuous cut planes moved to the branches of the ulnar and radial arteries at the end of the brachial artery, the distribution of the blood vessel bunch gradually became scattered again and the dispersion increased slightly.

With respect to the dispersion degree of the ulnar artery, it can be seen that only the initial proximal segment was relatively low, whereas the distribution of blood vessels in the middle and distal segments gradually dispersed. The cut planes with the dispersion of $\mathrm{X}$ and $\mathrm{Y}$ less than $6 \mathrm{~mm}$ were the 1 st to the 12 th, with a length of approximately $6 \mathrm{~cm}$. As for the radial artery, the dispersion degrees of $\mathrm{X}$ and $\mathrm{Y}$ were less than $6 \mathrm{~mm}$ from the 4 th cut plane to the 31 st cut plane, and the total length was approximately $13.5 \mathrm{~cm}$ (Fig. 3C-E).
A

B
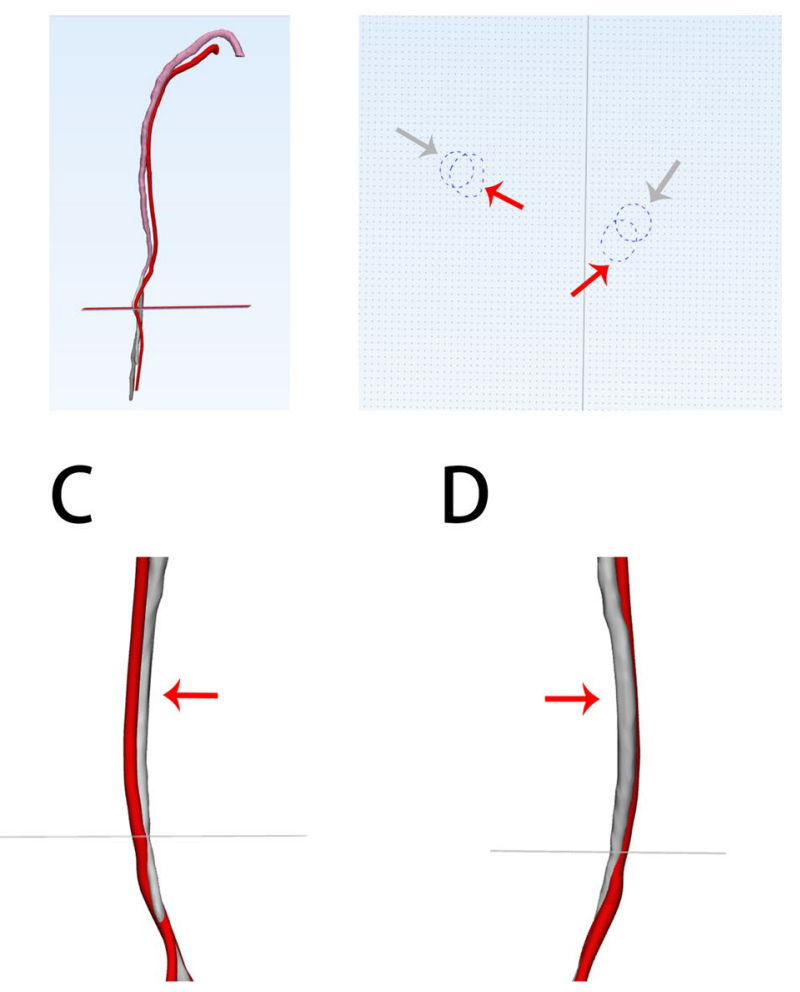

D

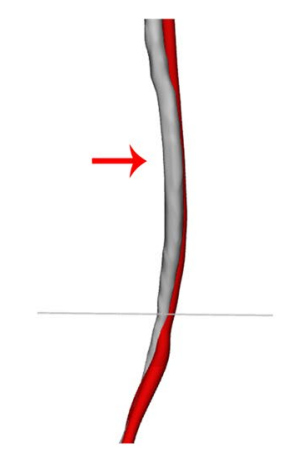

E
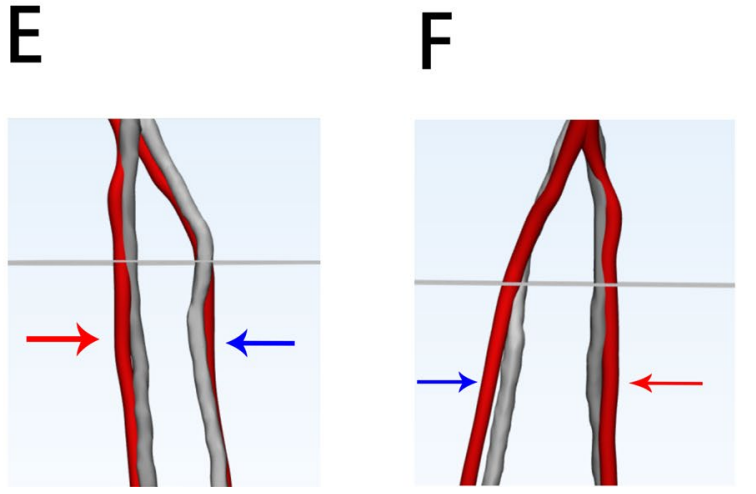

\subsection{The Standardized Blood Vessel Model was in Accordance with the Actual Blood Vessels}

As shown in Fig. 4A and B, the direction of the standard model and the actual blood vessel was basically the same, and the locations were close to or even coincided with each other. Figure 4C-F show the high accordance between the standard model of the brachial artery, the radial artery, and the ulnar artery and the actual blood vessel, especially the aforementioned low-dispersion segments. The results collectively show that the standard human blood vessel model was representative of the anatomical structure of 
human blood vessels and could have great potential for application in medicine.

\section{Discussion}

As the structural basis of blood circulation, blood vessels are closely associated with the occurrence and development of tumor and diverse relevant diseases [9]. Studies have shown that liver cancer, colorectal cancer, and breast cancer can induce blood vessels to extend into the tumor to provide nutrients [10-12]. The evaluation of blood vessel malformation and displacement can be crucially important for surgery and for follow-up with comprehensive therapy [13]. For blood vessel diseases, compared with the 2D planar image data, the 3D blood vessel reconstruction is of much higher accuracy in showing blood vessel structure [14]. It can provide a 3D visual impression in the diagnosis of coronary atherosclerosis, hemangioma, renal artery malformation, and other diseases, and potentially has the ability to decrease intraoperative bleeding, reduce contrast agent dosage, shorten the operative time, and decrease the radiation dose [15-17]. Whereas, thus far, the standardized blood vessel model, which can serve as an essential basis for evaluating 3D blood vessel structural changes and play a critical role in blood vessel disease diagnosis and surgical planning, has not been reported. In view of this, based on the 3D reconstruction, registration, and fitting of CTA images, the present study preliminarily proposed the methods of establishing a standardized blood vessel model.

To reduce the objective influence of somatotype difference on blood vessel structure, adult volunteers with the similar height, weight, and body mass index (BMI) were recruited. In addition, considering the potential differences in structure and location of blood vessels between different genders, only male volunteers were selected in this study to ensure that the standard model established was representative.

Because the CTA data of all eight subjects were in the arterial phase when their image data were collected, the CT value of the artery was significantly higher than that of the vein, nerve, and other surrounding soft tissues, which facilitated the division of the artery structure. As a result of the gap between adjacent cut planes in CTA scanning of artery and bone structure, a morphological interpolation method was used to ensure that the interested structure could be presented stereoscopically according to the $2 \mathrm{D}$ image data, relatively consistently with the real structure.

Registration was the basic step of establishing the standardized blood vessel model. Because the upper limbs of different subjects had different bending angles and rotation angles when collecting CTA data, and to make the bones and blood vessels of different volunteers calculable in a unified reference system, it was necessary to align the positions of the bones and blood vessels of the volunteers, that is to say, registration. In addition, because the larger arteries of the human body have relatively uniform trends of location and direction, it is also necessary to eliminate the errors caused by the flexion, extension, and rotation of the upper limbs through segmentation of bones and blood vessels.

It has been reported that active moving anatomical markers, such as the heart surface, or manually installed body surface markers were used as the marker points for registration [18-20]. In this study, we used the skeleton as the marker points to ensure that the registration results were more stable and accurate, because the skeleton had no position change during image acquisition. At the same time, there was no need to install additional artificial markers, thus simplifying the operation. In addition, in this study, the registration marker points were all determined manually by senior physicians, because compromising results have been reported after the software automatically selected the marker points for automatic registration [20]. Through the unified registration reference points on the skeleton, the skeleton and the corresponding blood vessels of different positions can be registered, by which the error caused by the limb angle and length can be eliminated, making the data of different volunteers comparable. The blood vessel bundle formed by registration will have a relatively uniform blood vessel direction, which has been delineated in the present study, forming the basis for further blood vessel fitting. For the same marker points, the larger the volume of the holistic matching part, the larger the matching error becomes. In our previous exploration experiment, the whole body skeleton had been registered as a whole with the upper limb skeleton providing the marker points for matching. However, great deviation was found at the shanks and feet, far away from the upper extremity, which undoubtedly could not provide guaranteed accuracy for further blood vessel fitting. In similar studies, there were also reports that the registration employing marker points far from the interested area became ineffective, necessitating manual recalibration [18]. In our study, we used segmental registration, which focused exactly on the local area. Utilizing the nearby bones, the segmented blood vessels of the upper limbs underwent precise registration, improving the accuracy of the subsequent standard blood vessel model fitting.

The processes of dispersion analysis and blood vessel fitting were the next core steps in creating a standardized blood vessel model. The calculation of blood vessel dispersion has been reported, but it was mainly focused on the micro-vessel; moreover, the degree of dispersion was largely estimated using pixel and image processing [21-23]. Therefore, in this study, in which we aimed to find the distance between the macro-vessel structure, it was innovative to use the integration method to accurately calculate the dispersion degree and 
fit the standard vessel model. In the present study, through calculation, it was found that the proximal and distal segments of the brachial artery had high degrees of dispersion, which were not suitable to serve as basic segments for standardized blood vessel model fitting. This high dispersion was particularly obvious in the proximal segments, which might be accounted for by the subclavian artery. During CTA data collection, raising or distaling the shoulder will significantly change the relative position relationship between the humeral head and the sternoclavicular joint, after which the arteries between those two will demonstrate completely different directions. Meanwhile, respiratory movement will also lead to the continuous change of thoracic cavity volume, leading to the relative displacement of the blood vessels close to the thoracic cavity and even motion artifact $[18,19,24,25]$. By instructing volunteers to take a unified posture for image acquisition, or using some simple fixing stents, this type of dispersion will be reduced. In addition, it is worth mentioning that most blood vessels in the human body will not have such a large, uncorrectable deviation due to the change of posture. Therefore, this method of establishing standard blood vessel model through segmentation, registration, and fitting was valuable and innovative for the evaluation of most of the blood vessels in the human body.

The accordance of the standard blood vessel model was evaluated by actual contralateral blood vessels. And, a high degree of coincidence between them was evident. In view of the symmetry of the blood vessel development in both upper limbs with respect to normal people, the satisfactory accordance has excellent medical value.

There were some limitations in this study. Initially, the calculated standard model can be more accurate and targeted by larger sample size and grouping the subjects by different genders and heights, and calculating the standard model respectively, rather than simply expanding the sample size and trying to use one model to represent all kinds of people. However, the grouping methods were not achievable due to the limited sample size, and the sample size was limited to the difficulty of recruiting healthy volunteers willing to undergo CTA with relatively consistent height, body shape and gender. Second, the blood vessel centerline has been reported to be employed for automatic blood vessel segmentation, while in the present study, the inefficient method of manual segmentation was still in use[20]. Third, the point cloud, iterative-closest-point matching algorithm, and semiautomatic rigid 3D registration have been shown to be capable of registration with promising results [19]. Instead, in this study, a simple point-to-point registration with three marker points was used, potentially causing some imprecision. Finally, the iterative method was demonstrated to be able to achieve optimization of registration through repeated calculation, which can serve as a complement in our follow-up study [26].

\section{Conclusion}

The present study showed that the human standard blood vessel model based on image registration and fitting technology has promising representativeness for blood vessel structures in the human body, and can further provide a fast, practical, and innovative model for the diagnosis and treatment of relevant diseases.

Acknowledgements The first three authors (Dinghao Luo, Junxiang Wu, Ning Wang) contributed equally to this manuscript. The authors declare that they have no conflict of interest. This study was supported by grants from National Natural Science Foundation of China (81301546), 3D Snowball Project of Shanghai Jiaotong University School of Medicine (GXQ202009), Clinical Research Project of Multi-Disciplinary Team, Shanghai Ninth People's Hospital, Shanghai JiaoTong University School of Medicine (201701003), Shanghai Clinical Medical Center (2017ZZ01023), Shanghai Municipal Key Clinical Specialty, the National Key R\&D Program of China (2016YFC1100600), General program of NSFC (81972058) and Shanghai Key Clinical Specialty Construction Project - Biomedical Materials (matching funds), Three-year Action Plan of Shenkang Development Center (SHDC2020CR2019B), Huangpu District Industrial Support Fund (XK2020009) and National Key Science and Technology Infrastructure of Translational Medicine (Shanghai) Open Project (TMSZ-2020-207).

Funding This study was supported by grants from National Natural Science Foundation of China (81301546), 3D Snowball Project of Shanghai Jiaotong University School of Medicine (GXQ202009), Clinical Research Project of Multi-Disciplinary Team, Shanghai Ninth People's Hospital, Shanghai JiaoTong University School of Medicine (201701003), Shanghai Clinical Medical Center (2017ZZ01023), Shanghai Municipal Key Clinical Specialty, the National Key R\&D Program of China (2016YFC1100600), General program of NSFC (81972058) and Shanghai Key Clinical Specialty Construction Project - Biomedical Materials (matching funds), Three-year Action Plan of Shenkang Development Center (SHDC2020CR2019B), Huangpu District Industrial Support Fund (XK2020009) and National Key Science and Technology Infrastructure of Translational Medicine (Shanghai) Open Project (TMSZ-2020-207).

Data Availability Not applicable.

Code Availability Not applicable.

\section{Declarations}

Conflict of interest The authors declare that they have no conflict of interest.

Open Access This article is licensed under a Creative Commons Attribution 4.0 International License, which permits use, sharing, adaptation, distribution and reproduction in any medium or format, as long as you give appropriate credit to the original author(s) and the source, provide a link to the Creative Commons licence, and indicate if changes were made. The images or other third party material in this article are included in the article's Creative Commons licence, unless indicated otherwise in a credit line to the material. If material is not included in the article's Creative Commons licence and your intended use is not permitted by statutory regulation or exceeds the permitted use, you will 
need to obtain permission directly from the copyright holder. To view a copy of this licence, visit http://creativecommons.org/licenses/by/4.0/.

\section{References}

1. Varinska, L., Gal, P., Mojzisova, G., Mirossay, L., \& Mojzis, J. (2015). Soy and breast cancer: Focus on angiogenesis. International Journal of Molecular Sciences, 16, 11728-11749.

2. Jing, B. Q., Ou, Y., Zhao, L., Xie, Q., \& Zhang, Y. X. (2017). Experimental study on the prevention of liver cancer angiogenesis via miR-126. European Review for Medical and Pharmacological Sciences, 21, 5096-5100.

3. Yeo, D. M., et al. (2015). Correlation of dynamic contrastenhanced MRI perfusion parameters with angiogenesis and biologic aggressiveness of rectal cancer: Preliminary results. Journal of Magnetic Resonance Imaging: JMRI, 41, 474-480.

4. Xie, L., Ji, T., \& Guo, W. (2017). Anti-angiogenesis target therapy for advanced osteosarcoma (Review). Oncology Reports, $38,625-636$.

5. Leaute-Labreze, C., Harper, J. I., \& Hoeger, P. H. (2017). Infantile haemangioma. The Lancet (London, England), 390, 85-94.

6. Braunagel, M., et al. (2018). Dynamic CTA in native kidneys using a multiphase CT protocol-potential of significant reduction of contrast medium. Academic Radiology, 25, 842-849.

7. Tuncay, V., et al. (2018). Non-invasive assessment of coronary artery geometry using coronary CTA. Journal of Cardiovascular Computed Tomography, 12, 257-260.

8. Ibukuro, K., Takeguchi, T., Fukuda, H., Abe, S., Tobe, K., \& Tagawa, K. (2013). Spatial relationship between the hepatic artery and portal vein based on the fusion image of CT angiography and CT arterial portography: The left hemiliver. AJR American Journal of Roentgenology, 200, 1160-1166.

9. Wang, L. H., et al. (2017). CTGF promotes osteosarcoma angiogenesis by regulating miR-543/angiopoietin 2 signaling. Cancer Letters, 391, 28-37.

10. Lu, Y., et al. (2017). MicroRNA-140-5p inhibits invasion and angiogenesis through targeting VEGF-A in breast cancer. Cancer Gene Therapy, 24, 386-392.

11. Yang, W., et al. (2017). In vivo inhibitory activity of andrographolide derivative ADN-9 against liver cancer and its mechanisms involved in inhibition of tumor angiogenesis. Toxicology and Applied Pharmacology, 327, 1-12.

12. Tankova, L., et al. (2019). Endorectal power Doppler ultrasonography is a reliable method for evaluation of rectal cancer angiogenesis. European Review for Medical and Pharmacological Sciences, 23, 1661-1667.

13. Chaichanyut, M., \& Tungjitkusolmun, S. (2016). Microwave ablation using four-tine antenna: Effects of blood flow velocity, vessel location, and total displacement on porous hepatic cancer tissue. Computational and Mathematical Methods in Medicine, 2016, 4846738
14. Cheezum, M. K., et al. (2017). Anomalous origin of the coronary artery arising from the opposite sinus: Prevalence and outcomes in patients undergoing coronary CTA. European Heart Journal Cardiovascular Imaging, 18, 224-235.

15. Zhang, J., Wang, Y., \& Geng, D. (2010). Intracranial epithelioid hemangioendothelioma: An unusual CTA finding in one case. British Journal of Neurosurgery, 24, 294-295.

16. Acar, G., et al. (2015). Relationship of neutrophil-lymphocyte ratio with the presence, severity, and extent of coronary atherosclerosis detected by coronary computed tomography angiography. Angiology, 66, 174-179.

17. Meng, X., Mi, Q., Fang, S., \& Zhong, W. (2015). Preoperative evaluation of renal artery anatomy using computed tomography angiography to guide the superselective clamping of renal arterial branches during a laparoscopic partial nephrectomy. Experimental and Therapeutic Medicine, 10, 139-144.

18. Vernikouskaya, I., Rottbauer, W., Seeger, J., Gonska, B., Rasche, V., \& Wohrle, J. (2018). Patient-specific registration of 3D CT angiography (CTA) with X-ray fluoroscopy for image fusion during transcatheter aortic valve implantation (TAVI) increases performance of the procedure. Clinical Research in Cardiology, 107, 507-516.

19. Nypan, E., Tangen, G. A., Manstad-Hulaas, F., \& Brekken, R. (2019). Vessel-based rigid registration for endovascular therapy of the abdominal aorta. Minimally Invasive Therapy \& Allied Technologies: MITAT, 28, 127-133.

20. von Spiczak, J., et al. (2018). Fusion of CT coronary angiography and whole-heart dynamic 3D cardiac MR perfusion: Building a framework for comprehensive cardiac imaging. The International Journal of Cardiovascular Imaging, 34, 649-660.

21. Righi, M., Locatelli, S. L., Carlo-Stella, C., Presta, M., \& Giacomini, A. (2018). Vascular amounts and dispersion of caliberclassified vessels as key parameters to quantitate 3D micro-angioarchitectures in multiple myeloma experimental tumors. Scientific Reports, 8, 17520.

22. Righi, M., Giacomini, A., Cleris, L., \& Carlo-Stella, C. (2013). (3) $\mathrm{D}$ [corrected] quantification of tumor vasculature in lymphoma xenografts in NOD/SCID mice allows to detect differences among vascular-targeted therapies. PLOS ONE, 8, e59691.

23. Righi, M., Giacomini, A., Lavazza, C., Sia, D., Carlo-Stella, C., \& Gianni, A. M. (2009). A computational approach to compare microvessel distributions in tumors following antiangiogenic treatments. Laboratory Investigation, 89, 1063-1070.

24. Qiu, L., Tan, H., Cheng, D., \& Shi, H. (2018). The incremental clinical value of cardiac hybrid SPECT/CTA imaging in coronary artery disease. Nuclear Medicine Communications, 39, 469-478.

25. Fyrdahl, A., et al. (2018). Pulmonary artery imaging under freebreathing using golden-angle radial bSSFP MRI: A proof of concept. Magnetic Resonance in Medicine, 80, 1847-1856.

26. Nandish, S., Prabhu, G., \& Rajagopal, K. V. (2017). Multiresolution image registration for multimodal brain images and fusion for better neurosurgical planning. Biomedical journal, 40, 329-338. 